
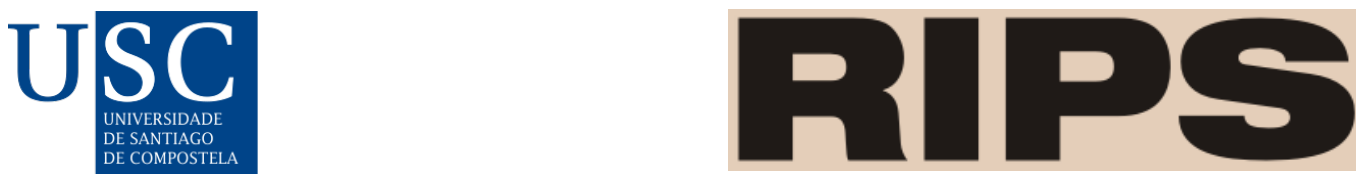

Revista

de Investigaciones

Políticas y Sociológicas

RIPS: Revista de Investigaciones Políticas y Sociológicas, 20(2), 2021. ISSN-e: 2255-5986

https://doi.org/10.15304/rips.20.2.7448

Artículos

\title{
La oposición parlamentaria en el Perú (2006-2020): de la cooperación al conflicto
}

The Parlamentary Opposition in Peru (2006-2020): From Cooperation to Conflict

\author{
Ignacio García Marín \\ Instituto Tecnológico de Monterrey, México \\ nachogarciamarin08@gmail.com
}

\section{Resumen}

La presente investigación trata de responder a la siguiente pregunta de investigación: ¿Cómo se han desarrollado las relaciones entre la oposición y el ejecutivo en el Perú para el período 2006-2020? Para ello, se realiza una aproximación cuantitativa en materia de actividad parlamentaria, composición del ejecutivo y un análisis del sistema de partidos peruano. En este sentido, este período es caracterizado por un marcado presidencialismo parlamentarizado y un elevado multipartidismo, se sucedieron tres mandatos presidenciales pero ocupados por seis jefes del Estado, dada la creciente inestabilidad y choque entre el ejecutivo y el congreso (unicameral). Se destaca la ausencia de coaliciones de gobierno y de mayorías oficialistas, así como la preeminencia de gabinetes apartidarios, lo que podría haber afectado la falta de compromiso por parte de los actores políticos. Asimismo, se identifican tres fases en cuanto a las relaciones entre la oposición y el oficialismo, evidenciándose una creciente hostilidad que finalizó con la situación de choque y bloqueo que caracterizó el bienio 2018-2020 y una grave crisis política.

Palabras clave: Perú; Congreso; Oposición; Ejecutivo; Coaliciones.

\begin{abstract}
This research analyzes the relations between the executive and the legislature in Peru in the period 2006-2020, characterized by a marked parliamentary presidentialism and a high degree of multipartyism. In this cycle, three presidential mandates were held by six heads of state, given the growing instability and clash between the executive and the congress (unicameral). The absence of government coalitions and official majorities, as well as the preeminence of non-partisan cabinets, could have affected the lack of commitment on the part of political actors. To this must be added the capacity of the opposition to condition the stability of the governments, including the head of state and the presidents of the council of ministers, but also the secondary role that the chamber took on in legislative matters. Likewise, three phases are identified in terms of the relations between the opposition and the ruling party, evidencing a growing hostility that ended with the situation of clash and blockade that characterized the 2018-2020 biennium and a serious political crisis.
\end{abstract}

Keywords: Peru; Legislative; Opposition; Executive; Coalitions. 


\section{INTRODUCCIÓN}

La oposición juega un rol de notable importancia en las democracias liberales, ya que pueden ser actores trascendentales para aprobar leyes, remover jefes del ejecutivo o dar estabilidad a los gobiernos. Asimismo, también les incumben tareas de representación, canalización de demandas sociales, control político o selección de diversos altos cargos. Por otra parte, el tratamiento que reciba la oposición por parte del Estado y, más en concreto del ejecutivo, puede ser un buen indicador para analizar la calidad democrática o la capacidad de inclusión de quienes gobiernan, donde el respeto y atención a las minorías es una cuestión crecientemente valuada a la hora de medir la representación de la pluralidad inherente a cada sociedad democrática. No deben ser ignorados en el análisis de un sistema político.

Su estudio, sin embargo, no ha sido desarrollado de la misma manera que otras áreas de la ciencia política, especialmente si se centra la atención en factores institucionales como la forma de gobierno o el sistema de partidos y la actividad legislativa. Mención aparte serían, no obstante, las investigaciones en torno a las coaliciones que se han desarrollado en los últimos años, destacándose las de Negretto (2006), Chasquetti (2008), Samuels y Shugart, (2010), Albala, (2016) y Araújo, Silva y Vieira (2016) entre otras. De igual modo, desde hace décadas se ha venido desarrollando una importante literatura en torno a las relaciones ejecutivo y legislativo, aunque no necesariamente centradas en la oposición como principal variable dependiente, sino más bien como actores necesarios para el estudio de los poderes políticos del Estado. Con respecto a la oposición parlamentaria, deben destacarse las investigaciones de Strøm (1984), Cheibub, Przeworki y Saiegh (2004); Morgenstern (2004); Morgenstern, Negri, y Pérez-Liñán (2009). Un punto que destacar es la propia pluralidad de lo que puede entenderse como oposición, la cual para la presente investigación se entiende como todo aquel grupo parlamentario no perteneciente al oficialismo, pero tampoco parte de una coalición pública y estable con el partido gobernante. En términos de Sartori, “oposición constitucional” (1966: p. 150).

\subsection{Objetivos y metodología}

Motivo de ello, la presente investigación se centra en el análisis de la oposición peruana y su capacidad de incidir en la actividad del Congreso y del ejecutivo, atendiendo a la siguiente pregunta de investigación: ¿Cómo se han desarrollado las relaciones entre la oposición y el ejecutivo en el Perú para el período 2006-2020? dado que un estudio de los últimos catorce años muestra interesantes resultados en cuanto a la producción legislativa, control político y estabilidad del gabinete. Para ello, se parte de un primer objetivo: el estudio de las principales características orgánicas del sistema político peruano, caracterizado por una parlamentarización de iure pero también de facto y una descripción comparada de las facultades constitucionales del presidente peruano. En segundo lugar, un estudio del sistema de partidos muestra una creciente fraccionalización del mismo, lo que ha terminado generando dificultades del ejecutivo para conformar mayorías en la cámara, pero también para mantener la estabilidad y la agenda del gobierno con independencia del legislativo. En tercer lugar, se intentan categorizar las distintas etapas que se identifican en cuanto a los efectos derivados de las relaciones entre los grupos opositores y el oficialismo, ya que los catorce años de estudio muestran una notable variación de 
estas, pero con una cierta tendencia al enfrentamiento entre poderes o incluso bloques políticos que habrían incidido en una desigual suerte y aplicación de las facultades constitucionales del ejecutivo y del legislativo.

Para lograr estos objetivos, se realiza un análisis con base cuantitativa, centrando la atención a indicadores habituales de la actividad parlamentaria y del sistema de partidos peruano, siguiendo a autores como Colomer \& Negretto (2005), Payne (2006), García Montero (2009) y Levitt (2012), así como de la propia composición del gabinete, dada su elevada inestabilidad y que el propio Levitt (2012) realizara para la época de los años 90.

En efecto, en este período, difícilmente se identifican coaliciones estables, públicas y de gobierno compartido, suponiendo a su vez una invitación a la categorización de estas relaciones y destacándose las consecuencias que habrían generado el sistema de partidos y el diseño institucional. Por ello, ha de subrayarse nuevamente el caso peruano, ya que grosso modo destaca por una institucionalización muy baja de su sistemas de partidos (Levitsky y Cameron, 2003; Chasquetti, 2008; Meléndez, 2012; Carter, 2018), así como por las mencionadas dificultades para la generación de coaliciones estables (Albala, 2009) y a que éstas se produzcan en todo caso de manera informal y breve, de manera similar a otros sistemas políticos de la región (Morgenstern, Negri y Pérez-Liñán, 2009; Albala, 2009). Todo ello ha ido acompañado de gabinetes con escasa composición partidaria (Corvetto, 2014), minorías oficialistas en el Congreso (unicameral) y a una potencial debilidad del ejecutivo ante la oposición, especialmente por los rasgos parlamentarizados del presidencialismo peruano.

Asimismo, y como adelantaron las investigaciones de Stepan y Skach, (1993), Mainwaring y Cox (1993) y Morgenstern y Wolfson, (2001), los incentivos tanto positivos como negativos para las relaciones entre el ejecutivo y el legislativo envuelven de utilidad a esta forma de gobierno como objeto de estudio. Dada la elección separada del jefe de Estado y de los legisladores, aparentemente el interés para llegar a acuerdos es menor, especialmente en aquellos sistemas políticos donde no se reconoce la posibilidad de la reelección inmediata. A ello súmese que la oposición puede entenderse como el conjunto de los perdedores de la contienda electoral y que no obtienen posiciones de gobierno a priori.

\section{EL PRESIDENCIALISMO PERUANO Y SU PARLAMENTARIZACIÓN: HERRAMIENTAS PARA LA INESTABILIDAD}

En efecto, el sistema político peruano cuenta con una serie de características propias que han de ser resaltadas. En primer lugar, la existencia del presidente del Consejo de Ministros o premier (PCM en adelante), el cual es nombrado por el jefe del Estado, pero requiere de una mayoría parlamentaria durante los primeros 30 días a la asunción de sus funciones. Esta figura, más propia de un régimen híbrido o del parlamentarismo, tiene sin embargo pocas facultades, estando supeditado al jefe del Estado en su acción y dedicándose especialmente a labores de coordinación y control dentro del consejo de ministros (Rubio Correa, 1999: 391). Puede ser cesado por moción de confianza perdida o por censura, así como por propia decisión del jefe del Estado (Arts. 132 y 133 de la CPP). La moción de confianza, además, no ha de ser constructiva, lo que puede agravar su dependencia parlamentaria. No obstante, el jefe de Estado requiere del refrendo ministerial para sus actos (art. 120, CPP), lo que reviste de cierta gravedad las crisis totales de gabinete. 
En segundo lugar, ha de destacarse una facultad presidencial no habitual en los regímenes políticos latinoamericanos: la disolución del congreso (art. 134 de la CPP). Esta potestad, a diferencia de la existencia del PCM, sí puede decirse que empodera al ejecutivo en detrimento del legislativo, dado que puede emplearse a partir del cese de dos PCM por parte del congreso en un mismo mandato presidencial y puede venir acompañada de una iniciativa legislativa, ofreciendo por tanto la posibilidad de chantaje o presión hacia los congresistas. No obstante, el cierre congresual no ha sido llevado a cabo desde 1993 (fecha de la actual constitución) salvo en el reciente caso de 2019 por parte de Martín Vizcarra, aunque es una herramienta que otros jefes del Estado han amagado con emplear en los últimos años (caso de Ollanta Humala). Pese a todo, la disolución debe dar lugar a elecciones legislativas en menos de cuatro meses, resultando electos parlamentarios que acabarán el mandato de sus pares anteriores, por lo que se mantiene la concurrencia electoral (art. 134 de la CPP). No puede ser empleada en los últimos doce meses del período presidencial.

Por otro lado, los mandatos presidenciales son de cinco años, con reelección ilimitada, pero no inmediata (art. 112 de la CPP), donde el legislativo es monocameral y está compuesto por 130 escaños desde 2011 (previamente por 120). El presidente de la república es acompañado por dos vicepresidentes, los cuales no cuentan con facultades especiales, siendo habitualmente actores marginales dentro del poder Ejecutivo. Esta información sin embargo ha de complementarse con otras variables, caso de un sistema de partidos muy poco institucionalizado (Tanaka \& Vera, 2010; Meléndez, 2012; Levitsky, 2018) y una arena política donde las principales instituciones del Estado han tenido recurrentes bajas valoraciones sociales. A este respecto, pueden destacarse las apreciaciones de autores como García Marín (2019), quien resalta la parlamentarización del presidencialismo peruano producida en los últimos años por la combinación de su diseño institucional, la capacidad de la oposición para determinar la estabilidad del ejecutivo y el bajo éxito legislativo de éste. García Belaunde (2009) lo caracteriza de presidencialismo atenuado, ya que en su opinión la figura del PCM acrecienta la capacidad de control por parte del legislativo hacia el ejecutivo, hecho que pudiera verificarse por la elevada rotación de ministros y primeros ministros desde 2001. García Montero (2009), haciendo referencia especialmente a las relaciones ejecutivo -legislativo, destaca igualmente la atenuación o debilitamiento de la figura presidencial. Levitt (2012: 4) en cambio, y en relación con el período 1990 - 2006, refiere de un hiperpresidencialismo, pues a pesar del diseño institucional, el sistema de partidos debilitó al congreso como contrapeso en un entorno de autoritarismo competitivo.

\subsection{Una aproximación comparada a las facultades del legislativo peruano}

Ahora bien, ¿qué tan poderoso es el legislativo del Perú? Dejando de lado las características anteriormente mencionadas, pueden subrayarse los estudios comparados que se han llevado a cabo a nivel latinoamericano Alcántara, García Montero y Sánchez (2005: 181) en su Índice de Capacidad Potencial de Control Político (en adelante, ICPCP), analizaron los legislativos latinoamericanos con base en diferentes facultades de control político al ejecutivo. En este sentido, otorgaron al congreso peruano la máxima puntuación de la región, destacándose la fortaleza del legislativo en sus facultades de moción de confianza y censura, así como las comisiones de investigación entre otras. Efectivamente, el congreso peruano está facultado para interpelar a cualquier miembro del consejo de ministros, lo que además puede venir secundado de una moción de censura si no 
resultó satisfecho por las explicaciones expuestas. Esto igualmente se puede derivar del discurso presidencial, ya sea directamente hacia el jefe del Estado (vacancia) o bien ante el PCM (moción de censura). De ahí, que en términos normativos pueda afirmarse que el congreso peruano tiene una notable capacidad de controlar y penalizar al ejecutivo, especialmente en lo que se refiere a su composición. Véase la siguiente tabla para una información más detallada:

Tabla 1. Resumen de valores desagregados del índice ICPCP

\begin{tabular}{|c|c|c|c|c|c|c|c|c|c|}
\hline & Comparecencia & $\begin{array}{l}\text { Com. } \\
\text { Investigación }\end{array}$ & $\begin{array}{l}\text { Discurso } \\
\text { Presid. }\end{array}$ & Confianza & Informes & Preguntas & Interpelaciones & Censura & Juicio \\
\hline Perú & $1 ’ 25$ & $1 ' 4$ & 1 & 1 & $1 ' 25$ & 0 & 1 & 3 & 1 \\
\hline Argentina & 1 & 1 & 1 & 0 & 1 & 0 & 1 & 3 & $1 ' 8$ \\
\hline Brasil & $1 ' 15$ & $1 ' 5$ & 1 & 0 & $1 ' 25$ & 0 & 0 & 0 & 1 '8 \\
\hline Chile & 1 & $1 ' 25$ & 1 & 0 & $1 ' 25$ & 1 & 0 & 0 & 1,8 \\
\hline Colombia & $1 ' 25$ & 0 & 1 & 0 & $1^{\prime} 25$ & 0 & 1 & 3 & $1 ' 8$ \\
\hline México & 1 & 1 & $1 ' 25$ & 0 & 1 & 0 & 0 & 0 & 1 '8 \\
\hline Uruguay & 1 & 1 & 1 & 1 & 1 & 0 & 1 & 2 & $1 ' 8$ \\
\hline Promedio & 1’06 & $0 ’ 99$ & 1'13 & $0{ }^{\prime} 1$ & 1 & 0,22 & $0 \times 77$ & $1 ’ 75$ & $1 ' 48$ \\
\hline
\end{tabular}

Fuente: elaboración propia con base en Alcántara et al. (2005: 181).

Esta información ha de ser complementada con las facultades legislativas del poder ejecutivo, especialmente en lo que se refiere a su capacidad y éxito en la proposición de proyectos de ley, así como en la emisión/revisión de decretos y el empleo del veto u observación. A este respecto, Payne (2006) sitúa al Perú en la parte medio-alta de la región, destacándose los poderes de decreto y de referéndum, facultad ésta última de muy bajo empleo por parte de los ejecutivos peruanos. Sí ha sido importante el uso del decreto, especialmente el referido a las cesiones legislativas, ya que de manera puntual el Congreso ha tendido a emitir leyes autoritativas que permitieran al ejecutivo legislar ante las dificultades de los grupos parlamentarios para alcanzar mayorías o bien en determinadas crisis económico-sociales, caso de la reciente pandemia por la Covid19.

\section{EL LEGISLATIVO PERUANO: DE LA INESTABILIDAD A LA HOSTILIDAD}

\subsection{El sistema de partidos peruano}

Con respecto al sistema de partidos peruano, ha de enfatizarse la ya mencionada baja institucionalización, característica que sigue la tónica de otras democracias latinoamericanas de nueva generación (Mainwaring y Scully, 1995; Mainwaring, 1999; Levitsky, Loxton y Van Dyck, 2016) y su creciente atomización, la cual es notable desde el año 2000 y que se combinó con una decreciente penetración territorial (Seifert Bonifaz, 2014), variación de las marcas partidarias (Levitsky, 2018), pero con clara permanencia de los candidatos en las elecciones presidenciales (García Marín, 2019) y un creciente rol que vienen jugando los outsiders y candidatos independientes en los últimos años (Meléndez, 2006; Carter, 2018). Su elevada fraccionalización así como el juego de alianzas y relaciones entre bancadas hace más adecuado que no se deba hablar de una oposición, sino de varias oposiciones.

A ello ha de sumarse una elevada volatilidad presidencial y parlamentaria, la cual se mostró en el mediocre desempeño de los oficialismos luego del fin de su mandato o la creciente necesidad de conformar coaliciones para lograr superar la valla electoral, actualmente en el 5\%. En este sentido, el aprismo pasó del 20.6\% de los votos en las elecciones parlamentarias de 2006 al $6.43 \%$ en 
2011, logrando la presidencia en 2006 pero no presentando candidato en 2011. En ese mismo año, la coalición Gana Perú de Humala logró el 25.27\% de los votos, no alcanzando un acuerdo para presentarse en 2016. Finalmente, Kuczynski (también conocido como PPK) obtuvo con su fórmula parlamentaria Peruanos Por el Kambio, el 16.46\% de los votos, no pudiendo presentar su sustituto, Vizcarra, la misma fórmula para las elecciones parlamentarias extraordinarias de 2020, aunque se apoyó en Somos Perú, quien recibió el 6.05\% de los votos válidos. El castigo al oficialismo saliente fue por tanto notable y rápido, así como la variación en la composición de los grupos parlamentarios a lo largo del tiempo:

Tabla 2. Evolución de las tres principales bancadas por elección y último período de sesiones, 2006-2020

\begin{tabular}{|c|c|}
\hline Elecciones parlamentarias de 2006 & $\begin{array}{l}\text { Unión por el Perú (37.5\%), APRA (30\%), Unidad } \\
\text { Nacional }(14.1 \%)\end{array}$ \\
\hline $\begin{array}{l}\text { Último período de sesiones de la legislatura } \\
\text { 2006-2011 }\end{array}$ & APRA (30\%), Nacionalista (19\%), UN (10\%) \\
\hline Elecciones parlamentarias de 2011 & $\begin{array}{l}\text { Gana Perú (36\%), Fuerza } 2011 \text { (28.4\%), Perú Posible } \\
(16.1 \%)\end{array}$ \\
\hline $\begin{array}{l}\text { Último período de sesiones de la legislatura } \\
\text { 2011-2016 }\end{array}$ & $\begin{array}{l}\text { Fuerza } 2011 \text { - Popular }(26.1 \%) \text {, Nacionalista - Gana Perú } \\
(20 \%) \text {, Dignidad y Democracia }(9.2 \%)\end{array}$ \\
\hline Elecciones parlamentarias de 2016 & $\begin{array}{l}\text { Fuerza Popular }(56.1 \%) \text {, Frente Amplio }(15.3 \%) \\
\text { Peruanos por el Kambio }(13.8 \%)\end{array}$ \\
\hline Elecciones extraordinarias de 2020 & $\begin{array}{l}\text { Acción Popular (19.2\%), Alianza para el Progreso } \\
\text { (16.9\%), Frente Popular Agrícola Fia del Perú }(11.5 \%) \text {, } \\
\text { Fuerza Popular }(11.5 \%)\end{array}$ \\
\hline
\end{tabular}

Fuente: elaboración propia con base en datos del Congreso de la República

Asimismo, el período analizado ha destacado por cierta polarización en las elecciones presidenciales, siendo las de 2011 y 2016 una clara pugna entre el fujimorismo y el anti fujimorismo en la segunda vuelta y por victorias muy ajustadas. A este respecto, el margen de victoria electoral se situó en el 5,26\% para las elecciones presidenciales de 2006; del 2.9\% para 2011; y de apenas $0.24 \%$ en 2016. Precisamente, el fujimorismo ha sido uno de los principales y más constantes movimientos políticos en el país, pues se caracterizó por una tendencia creciente en cuanto a resultados parlamentarios y a una cierta cohesión de su bancada, sólo igualada por el Aprismo y Acción Popular, aunque con grupos parlamentarios de menor tamaño. Sus resultados legislativos guardaron cierto paralelismo con las presidenciales, logrando 13 escaños en 2006, 37 en $2011^{1}$ y 73 en 2016. En las recientes elecciones parlamentarias extraordinarias de 2020 no lograron un resultado creciente, situándose en 15 luego de la crisis sucedida por la escisión liderada por Kenji Fujimori en 2018, el escándalo de corrupción en torno a Keiko Fujimori en 2019 y la posible interpretación del electorado peruano de que obstruyeron el proceso de reforma política.

Con respecto a las bancadas oficialistas, éstas han destacado por no lograr mayorías calificadas en el Congreso, especialmente en los últimos años analizados. Alan García se apoyó para su segundo mandato presidencial en una bancada que supuso el 30\% de la cámara, siendo el fujimorismo su principal aliado, con el 10.8\% (Meléndez, 2012). Humala contó con el 36\% de diputados, aunque tuvo escisiones a raíz de la conformación de dos nuevas bancadas y finalizó el quinquenio con el $20 \%$ de apoyos oficialistas. Kuczynski en cambió sólo pudo contar con $16.5 \%$ de los escaños, hecho que le situó desde un principio en debilidad ante el legislativo. Vizcarra, no obstante, fue el presidente que careció de mayores apoyos directos en la cámara, ya que al asumir como presidente apenas pudo contar con el 7.6\% de los curules y finalizó su mandato con un escaso $8.4 \%$ de los 
escaños que pueden considerarse oficialistas. El gobierno dividido ha sido una constante en los últimos años.

Por otro lado, esta debilidad partidaria con la que contaron los presidentes peruanos, así como la muy elevada inestabilidad de los grupos parlamentarios, dificulta la clara denominación de oposición y de aliados al oficialismo, ya que el transfuguismo, la baja cohesión de las bancadas y los débiles lazos programáticos e ideológicos en los partidos, facilitó un gran cambio en la composición en una parte considerable del arco parlamentario. En este sentido, es muy destacable la evolución de la composición del Congreso de la República. El número efectivo de partidos parlamentarios (NEPp en adelante) fue muy variable, pasando de 3.83 en el 2006, 3.97 en 2011, 2.76 en 2016 y 8,87 en las elecciones legislativas extraordinarias de 2020. A ello súmese la gran variación dentro de los quinquenios presidenciales, ya que fue relativamente habitual la escisión y conformación de nuevos grupos parlamentarios de manera anual para todo el período de estudio, como puede verse en el siguiente gráfico:

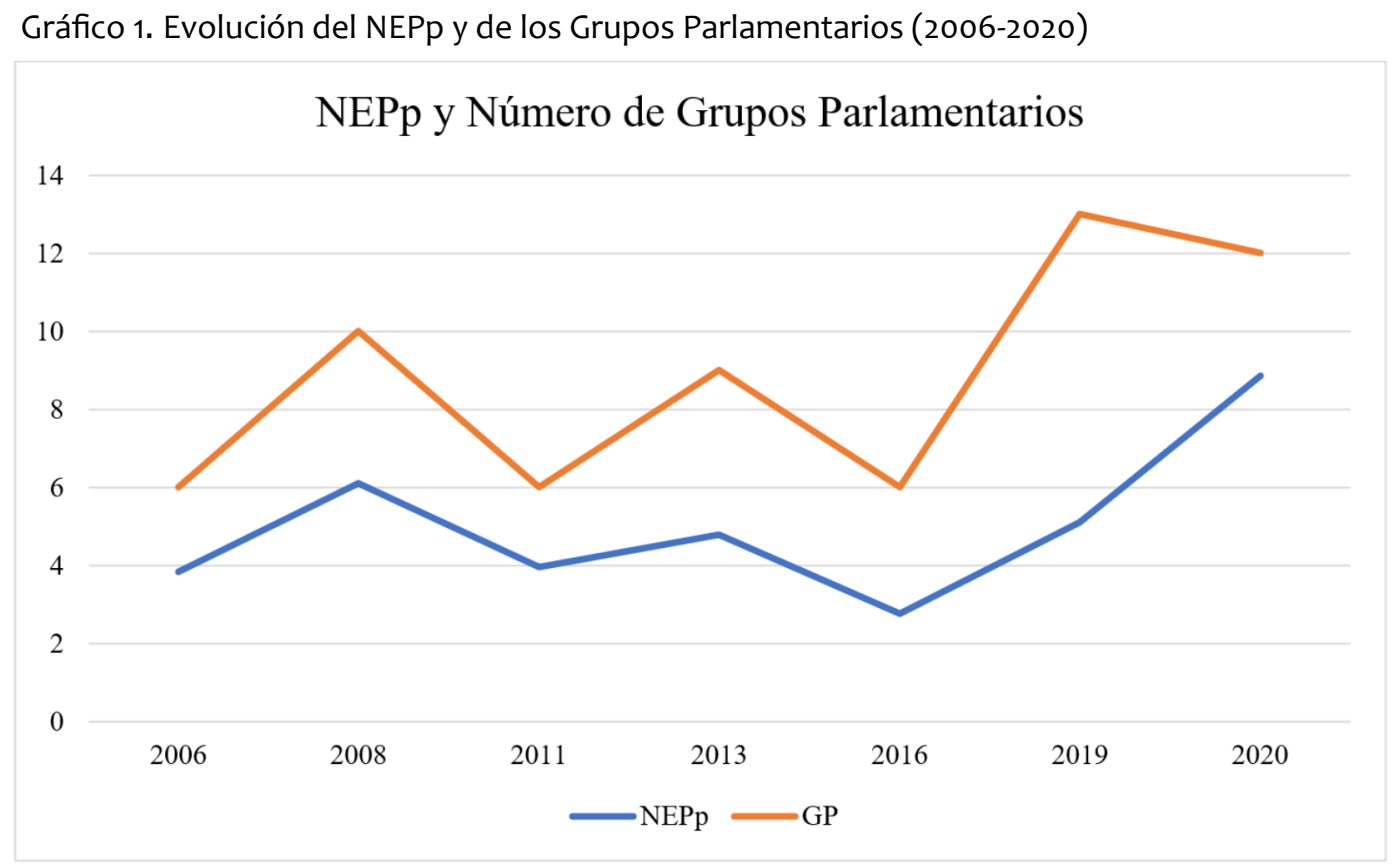

Fuente: elaboración propia con base en datos del Congreso de la República.

En efecto, la inestabilidad de los grupos parlamentarios ha sido una constante a lo largo de los catorce años analizados. Ya fuera por la volatilidad electoral o por la conformación de nuevas bancadas, lo cierto es que el paso de los períodos anuales de sesiones mostraron claras variaciones en la composición de los grupos. Por ejemplo, en 2006 iniciaron el período anual de sesiones 6 bancadas, pasando a ser 10 en el primer período de 2009-2010. En 2011 iniciaron 6, siendo ya 9 para el primer período de sesiones de 2013 - 2014. En 2016, se conformaron en un principio 6 grupos parlamentarios, para finalizar 2019 con 13. Tras las elecciones extraordinarias legislativas de 2020, se inició el período complementario con 12 bancadas. Las excepciones más claras a esta atomización fueron sin duda el aprismo y el fujimorismo, aunque con ciertas matizaciones, ya que el aprismo salvo en 2011 - 2016 contó con bancadas muy reducidas y el fujimorismo sufrió la inestabilidad y volatilidad de los demás grupos a partir de 2018. 


\subsection{La actividad legislativa y el choque hacia el ejecutivo}

La minoría oficialista en el Congreso de la República tuvo consecuencias para la relación de fuerzas hacia la oposición, logrando ésta última desestabilizar la composición de los gobiernos peruanos, tanto en lo que se refiere a los ministros como a primeros ministros y al propio jefe del Estado. De este modo, y a partir de la salida de Alan García de la presidencia, sus predecesores se caracterizaron por una relación de debilidad ante la cámara, manifestándose ésta en la rotación de los miembros del gobierno y en su decreciente producción legislativa. Así, la duración promedio de los PCM para el período 2006 - 2020 fue de apenas once meses, destacándose los siete premieres de Humala durante su quinquenio o los seis de Martín Vizcarra para dos años y medio. Los ministros corrieron una suerte similar, ya que su estancia se ubicó en diez meses y catorce días ${ }^{2}$. A ello ha de sumarse la creciente dificultad con la que contaron los PCM para poder lograr la confianza de la cámara, ya que una constante en los catorce años analizados fue la decreciente suma de apoyos legislativos según transcurrieron los períodos presidenciales, así como la frecuencia de censuras que conllevaron la salida de éstos. De nuevo, la excepción fue la segunda presidencia de García, ya que ninguno de sus premieres fue removido por el Congreso y en general contaron con cierto apoyo de la cámara a partir de la asunción del cargo. Sin embargo, Humala, Kuczynski y Vizcarra tuvieron que enfrentar la sustitución de varios de sus PCM por la oposición del Congreso, ya fuera por moción de censura o por pérdida de confianza. Éste fue el caso de uno de los siete PCM de Humala, uno de los dos de Kuczynski y de dos de los cinco de Vizcarra ${ }^{3}$. Asimismo, el apoyo promedio entre 2006 y 2020 que los premieres recibieron en la cuestión de confianza se situó en apenas el 57\% de la cámara ${ }^{4}$.

Esto a su vez se acompaña con que la elevada rotación de los ministros y primeros ministros no vino siempre aparejada por la moción de censura presentada y ganada por la oposición o como resultado de interpelaciones ${ }^{5}$, ya que fue práctica habitual durante estos mandatos que, o bien el jefe del Estado renovara el gobierno con vistas a una posible mejora en la valoración social de su gestión, o bien que se produjera por escándalos de corrupción o mala gestión. No obstante, a menudo el ejecutivo actuó con rapidez para cesar a un ministro ante la mera amenaza de moción por parte del congreso. 
Gráfico 2. Porcentaje de apoyos en las mociones de confianza a premieres (2006-2020)

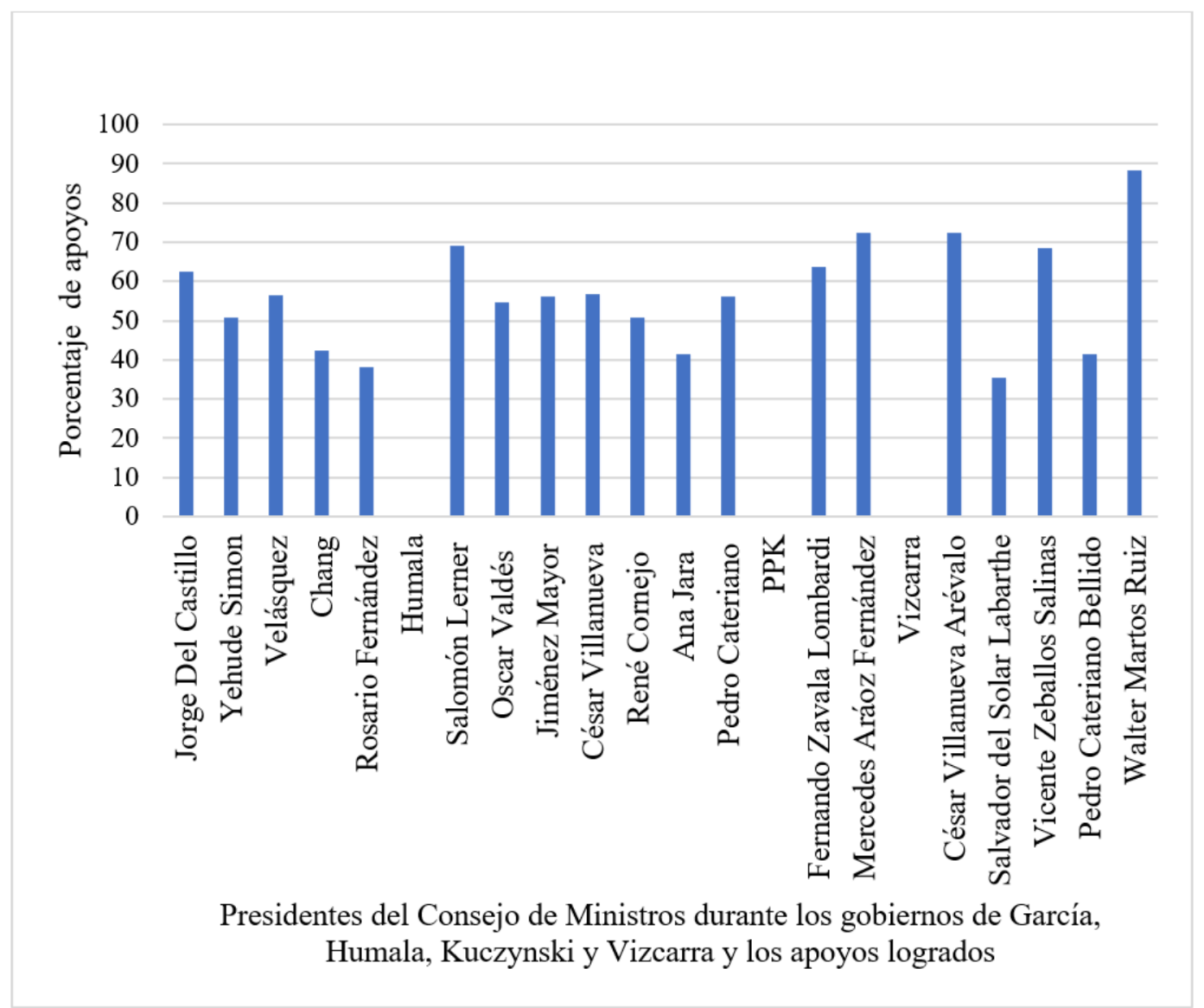

Fuente: elaboración propia con base en los datos del Congreso de la República.

Las medidas de control parlamentario no se centraron solo en las figuras secundarias del ejecutivo. Pedro Pablo Kuczynski en 2018 y Martín Vizcarra en 2020, tuvieron que enfrentar la vacancia presidencial o juicio político, terminando en ambos casos con su salida luego del inicio de investigaciones sobre posibles actos de corrupción generados antes de sus gobiernos, lo que sugiere una evidente debilidad ante el Congreso. Ambos presidentes contaban con escasos apoyos en el legislativo y en su contra con una oposición unida en este cometido, tanto a la izquierda como a la derecha del arco parlamentario. Por supuesto, esta minoría parlamentaria también se reflejó en la inestabilidad ejecutiva, pues sus PCM y ministros tuvieron la menor duración en promedio del período analizado.

Otra característica que debe ser subrayada es la ausencia de verdaderos gobiernos de coalición, así como de la baja afiliación partidaria de los ministros y primeros ministros, destacándose su carácter presuntamente tecnocrático, algo común en el Perú (Tanaka, Sosa y Puémape, 2016), así como en otros sistemas vecinos, caso de Ecuador (Garrido Rubio, 2003). Podría evidenciar la falta de atractivo de estos cargos para la negociación partidaria o bien, las dificultades de negociación por parte de los jefes de Estado hacia el legislativo. Esta falta de acuerdo y negociación exitosa es más llamativa si se recuerda la necesaria mayoría legislativa que el PCM requiere para su ejercicio. Así, mientras que durante el segundo gobierno de Alan García (2006-2011) el predominio fue claramente de miembros procedentes del aprismo y con algún ministro independiente en la 
recta final de mandato, en los gobiernos de Ollanta Humala (2011-2016), Pedro Pablo Kuczynski (2016-2018) y Martín Vizcarra (2018 - 2020) por debajo del 45\% de los ministros hizo pública su pertenencia al partido político del presidente o de terceros ${ }^{6}$. Esta falta de gobiernos integradores puede quizá explicar el porqué de su inestabilidad.

La legislación igualmente arroja datos e información reseñables. Aparentemente, pareciera haberse producido una cooperación selectiva y no muy frecuente entre el legislativo y el ejecutivo a lo largo del período de estudio, en concreto para los gobiernos de Kuczynski y Vizcarra. Las delegaciones de facultades legislativas en favor del ejecutivo tendieron a la baja, así como la emisión de decretos legislativos. No obstante, estas cesiones se produjeron bajo todas las presidencias, lo que sugiere un mínimo de cooperación y entendimiento entre ambos poderes. A este respecto, Alan García obtuvo en su segundo mandato cuatro cesiones para la emisión total de 142 decretos legislativos; Humala obtuvo siete cesiones para 131 decretos legislativos; Kuczynski únicamente una cesión para 111 decretos legislativos y Vizcarra cuatro cesiones para 101 decretos. En términos generales, las cesiones fueron destinadas a materias relacionadas con la seguridad y la economía, así como por la situación de excepcionalidad que la Covid19 generó a nivel nacional y mundial en materia de sanidad y economía durante el último año de mandato de Vizcarra.

Tabla 3. Producción legislativa en el período 2006-2020

\begin{tabular}{lccccc}
\hline Presidente & $\begin{array}{c}\text { Decretos } \\
\text { legislativos } \\
\text { (a) }\end{array}$ & $\begin{array}{c}\text { Decretos de } \\
\text { urgencia (a) }\end{array}$ & $\begin{array}{c}\text { Leyes } \\
\text { publicadas } \\
\text { origen } \\
\text { Congreso }\end{array}$ & $\begin{array}{c}\text { Leyes } \\
\text { publicadas } \\
\text { origen } \\
\text { Ejecutivo }\end{array}$ & Total \\
$\begin{array}{l}\text { García (2006- } \\
\text { 2011) }\end{array}$ & $\begin{array}{c}4 \text { cesiones } \\
142 \text { decretos }\end{array}$ & 383 & 393 & 288 & $\mathbf{1 2 0 6}$ \\
$\begin{array}{l}\text { Humala } \\
(2011-2016)\end{array}$ & $\begin{array}{c}7 \text { cesiones, } \\
131 \text { decretos }\end{array}$ & 53 & 192 & 273 & $\mathbf{6 4 9}$ \\
$\begin{array}{l}\text { Kuczynski } \\
(2016-2018\end{array}$ & $\begin{array}{c}1 \text { cesión, } 111 \\
\text { decretos }\end{array}$ & 20 & 220 & 51 & $\mathbf{4 0 2}$ \\
$\begin{array}{l}\text { Vizcarra } \\
(2018-2020)\end{array}$ & $\begin{array}{c}4 \text { cesiones, } \\
101 \text { decretos }\end{array}$ & 179 & 33 & 119 & $\mathbf{4 2 6}$ \\
Total & $\mathbf{4 8 5}$ & $\mathbf{6 3 5}$ & $\mathbf{8 3 8}$ & $\mathbf{7 3 1}$ & $\mathbf{2 6 8 3}$ \\
\hline
\end{tabular}

Fuente: (a) Tanaka y Vera (2010: 101) para datos hasta 2011; 2011-2020 elaboración propia con base en el Congreso de la República. No se consideran resoluciones legislativas ni otros actores proponentes ajenos al Congreso y Ejecutivo.

En cuanto a la producción de leyes, se observa una clara tendencia a la baja en cuanto al rol del ejecutivo, el cual quizá por una predominante minoría parlamentaria fue encontrando mayores dificultades para lograr sacar adelante sus proyectos de ley o bien tendió a desistir de iniciarlos. Si a las leyes se le añade la emisión de decretos, la bajada en cuanto a producción legislativa es más acusada. Es reseñable a su vez que el Congreso vivió un éxito variable a lo largo de los años, fruto de la atomización del sistema de partidos y de su claro reflejo en la inestabilidad de los grupos parlamentarios: 
Gráfico 3. Leyes publicadas por origen y por período anual de sesiones parlamentarias, 2006-2020

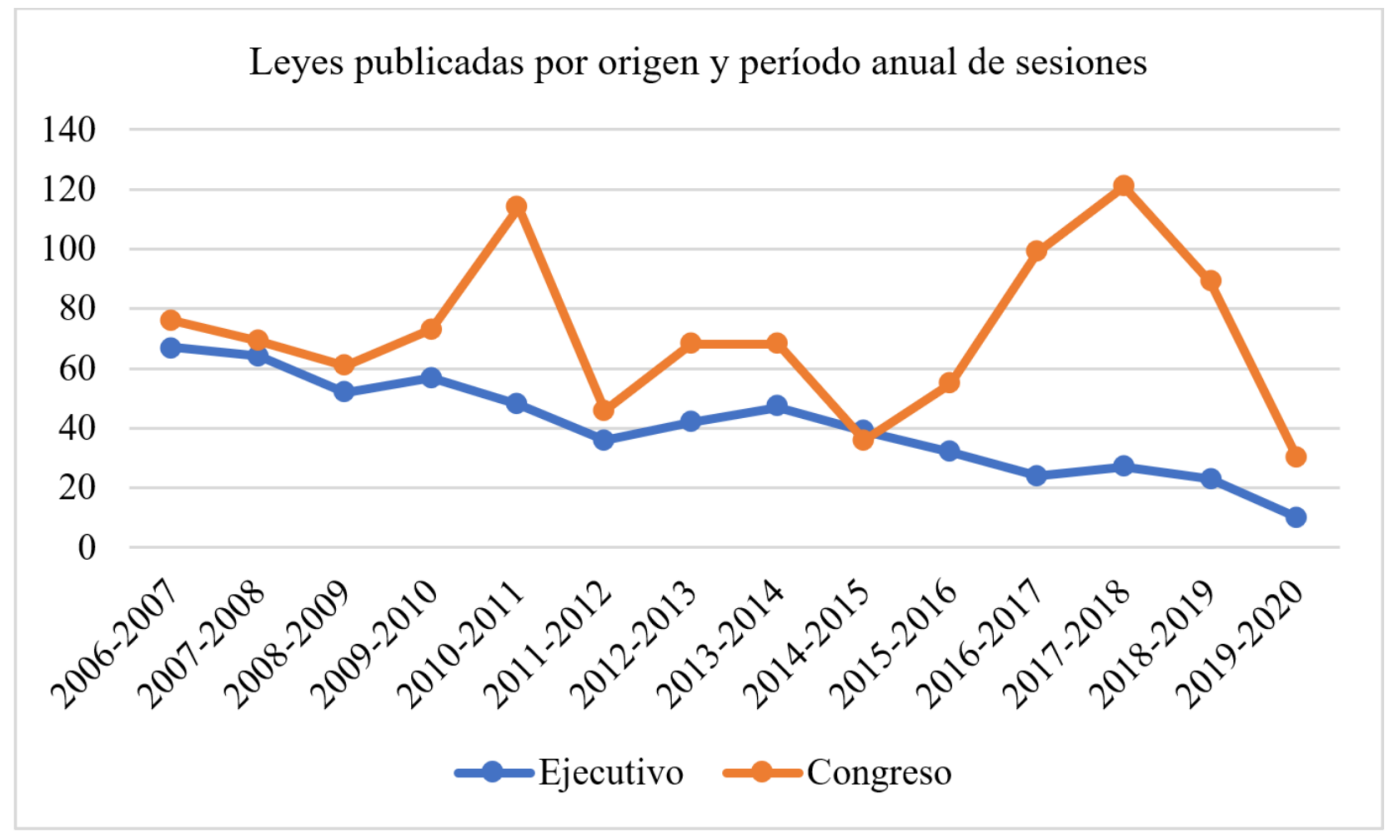

Fuente: elaboración propia con base en el Congreso de la República. Se excluyen resoluciones y mociones.

El comportamiento partidario pudo tener incidencia en la producción legislativa. Presidentes con mayores apoyos en el Congreso, como García y Humala, tendieron a apoyase en el legislativo para proponer proyectos de ley. En el caso del presidente aprista, destaca además la inflación legislativa de su período, muy superior al de sus predecesores. En cambio, Kuczynski y Vizcarra se encontraron en situación de marcada minoría parlamentaria, lo que les pudo llevar a emplear el decreto de manera alternativa a la iniciativa legislativa, tesis que entroncaría con las investigaciones de Cox, Morgenstern y Wolfson (2001) para los regímenes presidencialistas latinoamericanos.

\section{LA OPOSICIÓN PARLAMENTARIA EN EL PERÚ Y UNA POSIBLE INTERPRETACIÓN}

Como se ha podido observar, el legislativo peruano ha tenido la capacidad de condicionar la composición y estabilidad de los ejecutivos -especialmente durante las presidencias de Kuczynski y Vizcarra- dada la preeminencia del gobierno dividido y de la ausencia de ejecutivos integradores. La elevada rotación de ministros y primeros ministros ha sido muy acelerada, la cual vino además acompañada por la salida prematura de los dos últimos jefes de Estado, ambos con escasos apoyos partidarios en el Congreso y con una frontal y bien estructurada oposición fujimorista. En cambio, ha de recordarse que se evidenció una puntual y escasa cooperación entre el ejecutivo y el congreso, ya que las delegaciones de facultades en materia legislativa se produjeron a lo largo de los catorce años analizados, en línea a lo señalado por Negretto (2006) para el propio caso del Perú y en un período previo. Sin embargo, a diferencia de lo que muestran las investigaciones de Colomer y Negretto (2005: 81), el caso peruano no ilustra una cooperación más amplia entre poderes a pesar de la confianza parlamentaria que el PCM requiere para su ejercicio, a diferencia de lo observado en otros sistemas políticos de la región. Es decir, más allá de estos acuerdos puntuales, la oposición 
y el oficialismo no mantuvieron una colaboración y acuerdo de manera destacable a lo largo del período de estudio. De hecho, estas cesiones legislativas, así como la emisión de decretos, pueden entenderse igualmente como una manera de superar las dificultades del Congreso para legislar en un entorno de elevada y variable fraccionalización, siendo cada vez mayor la proporción de decretos en relación con la producción de leyes. Esto, de hecho, conecta con las tesis de Reniu y Albala (2012) para Argentina, Chile y Uruguay, pues evidenciaron que los gobiernos divididos tendían a acrecentar el uso del decreto en detrimento de la negociación legislativa e incentivándose el bloqueo entre poderes.

Por otro lado, el gobierno peruano se sitúa entre los más horizontales de la región (Araújo, Silva y Vieira, 2016), dada la existencia de un primer ministro con obligada confianza parlamentaria y el necesario refrendo ministerial de los actos del jefe del Estado (art. 120, CPP), lo que ha de ser acompañado con las facultades de control político del congreso peruano, como se mostró previamente. Por tanto, la muy elevada rotación y salida de miembros del consejo de ministros puede alterar la capacidad de maniobra del ejecutivo, rebajándose la separación rígida entre ambos poderes. Asimismo, el predominante carácter no partidario de los gobiernos peruanos bajo este entorno institucional y de minorías oficialistas, podría explicar parcialmente la inestabilidad de éstos y su escaso éxito a la hora de conformar coaliciones parlamentarias, hecho ya muy analizado por la literatura especializada para otros regímenes presidencialistas (Mainwaring 1993; Linz y Stepan, 1994; Samuels y Shugart, 2010; Chaisty, Cheeseman y Power, 2012). Precisamente, el Perú se sitúa como uno de los sistemas políticos con menor presencia partidaria en los gabinetes y donde los presidentes tienen menor capacidad o intención de emplear los puestos de gobierno como producto de negociación y consolidación de coaliciones parlamentarias, muy lejos de lo sucedido igualmente en otros sistemas de la región (Freudenreich, 2016). Dicho de otro modo, la parlamentarización del presidencialismo peruano y su sistema de partidos de elevada fraccionalización, así como la carencia de mayorías oficialistas y de coaliciones y la falta de compromisos a medio plazo entre oficialismo y oposición, podría explicar la inestabilidad y escasa duración de los ministros y primeros ministros.

Otro punto importante para destacar es que la disolución congresual que Vizcarra llevó a cabo en 2020 no fue suficiente para desbloquear o reducir la creciente tensión que existía entre el ejecutivo y el legislativo. Esta elección no resolvió el conflicto entre poderes, pues aun conformando la cámara con nuevos diputados (sólo cuatro lograron la reelección) y una nueva relación de fuerzas, fue cesado de su cargo en noviembre de 2020. Esta facultad constitucional, propia de los regímenes parlamentarios y que idealmente se entiende como una de las vías que se pueden emplear para la generación de nuevos actores y mayorías en el congreso, a fin de que pueda desbloquearse una situación de parálisis institucional, no logró el mencionado objetivo. Esta situación es de hecho más llamativa si se tiene en cuenta que la bancada que mostró mayor reactividad al ejecutivo, caso del fujimorismo, salió notablemente disminuida del proceso legislativo extraordinario de $2020^{7}$.

Por otro lado, puede decirse que se identifican tres etapas diferentes en cuanto a las relaciones oposición - oficialismo en el Perú. Una primera etapa de moderada cooperación, situada entre 2006 y 2011, en la que se produjo una coalición estable (aprismo y fujimorismo ${ }^{8}$ ), cesiones legislativas variadas, ausencia de mociones de censura y creciente protagonismo del ejecutivo en la creación de legislación. Es destacable que las dos principales bancadas fueron a su vez los de mayor institucionalización y cohesión interna, a pesar de compartir los elementos personalistas de las demás fuerzas peruanas. Los gabinetes de García fueron a su vez los de mayor carácter político, en 
especial los primeros ministros, teniendo tres de los cinco que ocuparon el cargo adscripción a una organización partidaria.

En una segunda etapa, coincidente con el mandato de Ollanta Humala (2011-2016), se identifica una cooperación menor, caracterizada por una moción de censura ganada por la oposición ante la PCM Ana Jara, así como varias planteadas o con la amenaza de ello ${ }^{9}$; un abierto enfrentamiento entre el oficialismo y el fujimorismo y una decreciente cooperación legislativa, dado que más de la mitad de los decretos emitidos en el quinquenio se produjeron en los dos primeros años de mandato. Esta moción de censura visibilizó no sólo la minoría parlamentaria del jefe del Estado, si no además la capacidad de la oposición para reaccionar y no otorgar la confianza a aquellos premieres que no fueran previamente consensuados con ellos. Humala enfrentó además una pérdida de miembros en su propia bancada. Primeramente, por la salida de diputados de izquierda que conformaron junto a otros el grupo parlamentario Acción Popular - Frente Amplio y, posteriormente, otra descomposición que dio lugar a Dignidad y Democracia. El debilitamiento del oficialismo fue claro en términos cuantitativos: de 47 escaños sobre 130 en el inicio del primer período legislativo a 26 en el último de ellos. Es decir, de la bancada inicial se terminaron conformando tres. Por ello, su creciente debilidad parlamentaria se acompañó de decreciente protagonismo legislativo y de una oposición más activa y exitosa en cuanto a control político y éxito legislativo. Es reseñable además que el PNP, al igual que el aprismo luego del fin de la segunda presidencia de García, no fue capaz de presentar candidato a la jefatura del Estado tras el fin del mandato de Humala.

Esta etapa fue un preludio de lo que sucedió en el siguiente quinquenio, caracterizado por contar con cuatro presidentes ${ }^{10} \mathrm{y}$ una abierta hostilidad entre Congreso y Ejecutivo, ya que Kuczynski fue removido de su cargo apenas veinte meses después de su toma de posesión, siendo su sustituto Vizcarra, también presidente durante solo treinta y dos meses. Su participación legislativa tendió a la baja en relación con los presidentes previos, aunque PPK llegó con una agenda económica en sintonía con el fujimorismo y Vizcarra recabó varios apoyos legislativos ante la crisis sanitaria y económica derivada de la pandemia. Sin embargo, su debilidad parlamentaria y la abierta hostilidad de la cámara les dejó en riesgo de enfrentar una salida anticipada, hecho que sucedió y que además se acompañó por una interpretación discutible, por parte de Vizcarra, de su facultad constitucional de disolver la cámara, lo que conectaría con la visión de Cox, Morgenstern y Wolfson (2001) sobre la tendencia a ampliar los poderes constitucionales por parte de presidentes débiles ante el legislativo; o con las primigenias tesis de Linz (1990; 1999), Mainwaring (1993), Linz y Valenzuela (1994) y Mainwaring y Shugart (1997) sobre los riesgos de los presidencialismos con sistemas partidarios altamente fraccionalizados.

La democracia peruana no ha contado con quiebres desde la salida de Fujimori, aunque lo cierto es que las protestas multitudinarias de noviembre de 2020 y el propósito obstruccionista de la oposición en los últimos meses de Vizcarra, han generado fuertes tensiones al sistema político, así como una notable crisis de legitimidad. De hecho, esta hostilidad o confrontación entre poderes puede encajar con la idea central de Hiroi y Renno (2014) en cuanto al peso del oficialismo, su capacidad de crear coaliciones parlamentarias y su relación con la producción legislativa y la actitud obstruccionista del congreso. En el caso del Perú, es claro el posicionamiento crecientemente hostil de las oposiciones según transcurren los quinquenios, especialmente destacable a partir de la presidencia de García y visualizado en las dificultades del ejecutivo para sacar adelante sus proyectos de ley y en mantener la confianza de sus premieres. 
No puede decirse por tanto que los gobiernos peruanos hayan logrado exitosamente imitar el estilo parlamentario de gobiernos de coalición, siguiendo la denominación de Chasquetti (2001) y Lanzaro (2001). Sin embargo, esta dinámica es similar a la observada para el Perú y otros Estados de la región en los años 80 y 90 (Morgenstern, Negri, y Pérez-Liñán, 2009) y que igualmente converge con las tesis de Strøm (1984), Zelaznik (2002) y Albala (2016): gobiernos con minoría parlamentaria y sin dar entrada a otras fuerzas partidarias en su gabinete. No obstante, no es menos cierto que la tendencia al establecimiento de gobiernos de coalición en América Latina ha sido creciente y estable, tal y como señalan las investigaciones de Dehesa (1997), Lanzaro (2001) y Cheibub, Przeworski y Saiegh (2004). El Perú, en todo caso, ha destacado por una acusada inexistencia de mayorías oficialistas y de coaliciones parlamentarias, así como por los efectos esperados que esta combinacion puede generar en sistemas parlamentarizados y sin gobiernos integradores.

\section{CONCLUSIONES}

El sistema político peruano destaca por la incorporación de elementos propios de un régimen parlamentarizado, caso de la existencia de un presidente del consejo de ministros o de la capacidad de disolución parlamentaria por parte del jefe del Estado. Sin embargo, estos incentivos, que $a$ priori debieran propiciar un mayor compromiso entre oposición y ejecutivo en la tarea de gobierno, no han surtido efecto. Muy al contrario, no puede hablarse de un gobierno compartido ni de una negociación exitosa entre los diferentes actores. Asimismo, se destaca un sistema de partidos de muy baja institucionalización, creciente fraccionalización y predominio de los candidatos sobre las organizaciones partidarias. Esto vino acompañado por la persistencia de las minorías oficialistas y la ausencia de coaliciones parlamentarias y de gobierno, confrontando con las tesis de Dehesa (1997), Cheibub, Przeworski y Saiegh (2004) y de Albala (2016) y en línea con lo que ya adelantaran Linz (1990; 1999) y Linz y Valenzuela (1994).

El resultado ha sido claro: gobiernos inestables que han sufrido constantes renovaciones, decreciente producción legislativa y una oposición reactiva cuando lo ha considerado, mientras el uso del decreto ganó protagonismo. Es decir, aunque la cooperación ha sido infrecuente, es observable, especialmente en materia de elección de premieres y de cesiones legislativas. El limitado compromiso de las oposiciones en materia de gobierno y legislación se situaría en línea a las investigaciones de Morgenstern, Negri y Pérez-Liñán (2009: 21). Otro hecho destacable es que, si bien es cierto que la existencia de gobiernos divididos y ausencia de coaliciones son relativamente frecuentes en América Latina, los efectos producidos por esta combinación distan de ser comparables. El diseño institucional y la cultura política, pudieran ser explicaciones plausibles a este distanciamiento.

Estas relaciones entre el ejecutivo y la oposición sugieren no obstante identificar tres diferentes etapas con base en lo observado. Una primera durante la segunda presidencia de Alan García (2006-2011), donde el oficialismo trazó una alianza sin entrada en el gobierno con el fujimorismo, lo que le permitió cierta estabilidad y dominio de la creación legislativa. García no se tuvo que enfrentar a numerosas interpelaciones ni a ninguna moción ni vacancia presidencial. Su grupo parlamentario mantuvo la composición, hecho a destacar para el sistema peruano, siendo su gobierno el de menor carácter tecnocrático. Posteriormente, con Ollanta Humala (2011-2016), el fujimorismo se configuró como la verdadera oposición, ayudada por la menguante minoría de 
Gana Perú y de un gobierno que encontró muchas dificultades para acordar con el parlamento una acción de gobierno. Su protagonismo legislativo decreció del mismo modo que aumentó la amenaza y ejecución de mociones de censura y la salida anticipada de ministros y primeros ministros. Además, el Congreso sufrió una acusada atomización de las bancadas. En tercer lugar, durante las presidencias de Kuczynski (2016-2018) y Vizcarra (2018-2020), el choque entre oposiciones y ejecutivo fue claro. El fujimorismo se ubicó como la primera fuerza del congreso, y nuevamente se quedó cerca de la presidencia. Ambos presidentes abandonaron prematuramente el poder, la sustitución de los primeros ministros fue la más acelerada del período analizado y se produjo la primera disolución congresual desde 1993. Tanto PPK como Vizcarra tendieron a legislar mediante decreto ante su incapacidad de alcanzar mayorías parlamentarias y por supuesto no lograron conformar coaliciones de gobierno. A eso se une la actitud obstruccionista de parte de la oposición ante una serie de reformas políticas defendidas desde la presidencia.

Debe decirse en cambio, que la falta de coaliciones y la persistencia del gobierno dividido no ha supuesto el fin del régimen democrático, aunque sí ha devenido en crisis políticas de cierta magnitud, donde la elevada fraccionalización pareciera haber debilitado a ambos poderes, con un congreso que se ha ido situando en un segundo plano y un ejecutivo débil e inestable. Por ello, puede decirse que al comparar los tres períodos presidenciales se evidencie que la existencia de coaliciones de gobierno sea un buen remedio a los hipotéticos incentivos negativos del presidencialismo, siguiendo la línea de Chasquetti (2006) y con base en la segunda presidencia de García (2006-2011).

\section{Referencias Bibliográficas}

ALBALA, Adrián. (2009). Coaliciones gubernamentales y régimen presidencial: incidencia sobre la estabilidad política, el caso del Cono Sur (1983-2005). Documentos CIDOB América Latina(29).

ALBALA, Adrián. (2016). Presidencialismo y coaliciones de gobierno en américa latina: Un análisis del PaPel de las institUciones. Revista de Ciencia Política, 459-479.

ALCÁNTARA, Manuel, GARCÍA MONTERO, Mercedes, \& SÁNCHEZ LÓPEZ, Francisco. (2005). Funciones, procedimientos y escenarios: un análisis del poder legislativo en América Latina. Salamanca: Ediciones Universidad de Salamanca.

ALEMÁN, Eduardo, \& TSEBELIS, George. (2012). Partidos Políticos y Coaliciones de Gobierno. Política. Revista de Ciencia Política, 50(2), 5-32.

ARAÚJO, V., SILVA, T., \& VIEIRA, M. (2016). Measuring Presidential Dominance over Cabinets in Presidential Systems: Constitutional Design and Power Sharing. Brazilian Political Science Review, 10(2).

CARTER, Christopher (2018). Party system erosion: evidence from Peru. Party Politics, 20(10), 1-13.

CECILIA, Martínez Gallardo. (2014). Designing Cabinets: Presidential Politics. Journal of Politics in Latin America, 6(2), 3-38.

CHAISTY, Paul, Cheeseman, Nic, \& Power, Timohty. (2012). Rethinking the 'presidentialism debate': conceptualizing. Democratization, 1-25.

CHASQUETTI, Daniel (2001). Democracia, multipartidismo y coaliciones en América Latina: evaluando la difícil combinación. En J. Lanzaro, ipos de Presidencialismo y Coaliciones Políticas en America Latina. (págs. 319-359). Buenos Aires: CLACSO.

CHASQUETTI, Daniel. (2006). La supervivencia de las coaliciones presidenciales de gobierno en América Latina. Postdata(11), 163-192. 
CHASQUETTI, Daniel. (2008). Democracia, presidencialismo y partidos políticos en América Latina: evaluando la difícil combinación. Montevideo: Ediciones Cauces.

CHEIBUB, José Anronio, PRZEWORSKI, Adam, \& SAIEGH, S. M. (2004). GovernmentCoalitions and Legislative Success under Presidentialism and Parliamentarism. British Journal of Political Science, 34, 565-587.

COLOMER, Josep Maria, \& NEGRETTO, Gabriel. (2005). Can Presidentialism Work Like Parliamentarism? Government and Opposition, 40(1), 60-89.

CORVETTO, Piero. (2014). Gobiernos sin partido: el reclutamiento de personal en la relación entre el gobierno y el partido de gobierno en el Perú. Revista De Ciencia Política Y Gobierno, 1(1), 11-36.

COX, Gary, MORGENSTERN, Scott, \& WOLFSON, Leandro. (2001). Legislaturas reactivas y presidentes proactivos en América Latina. (I. d. Social, Ed.) Desarrollo Económico, 41(163), 373-393.

DEHESA, Guillemo. (1997). Gobiernos de Coalición en el Sistema Presidencial: América del Sur. Tesis doctoral. Florencia: European University Institute.

FREUDENREICH, Jürgen. (2016). The Formation of Cabinet Coalitions in Presidential Systems. Latin American Politics and Society, 80-102.

GARCÍA BELAUNDE, Domingo. (2009). El presidencialismo atenuado y su funcionamiento. Con referencia al sistema constitucional peruano. En A. Ellis, J. J. Orozco Henríquez, \& D. Zovatto, Cómo hacer que funcione el sistema presidencial. Ciudad de México: UNAM.

GARCIA MARIN, Ignacio. (2019). Presidencialismo y sistema de partidos: la parlamentarización peruana (2001-2016). Espiral, Estudios sobre Estado y Sociedad(74).

GARCÍA MONTERO, Mercedes. (2009). Presidentes y Parlamentos: ¿quién controla la actividad legislativa en América Latina? Madrid.: CIS.

GARRIDO RUBIO, Antonio. (2003). Gobiernos y estrategias de coalición en democracias presidenciales: el caso de América Latina. Política y Sociedad, 40(2), 41-62.

HIROI, Taeko., \& RENNO, Lucio. (2014). Hiroi, T., \& Renno, L.Dimensions of Legislative Conflict: Coalitions, Obstructionism, and Lawmaking in Multiparty Presidential Regimes. Legislative Studies Quarterly, 39(3), 357-386.

LANZARO, Jorge. (2001). Tipos de presidencialismo y modos de gobierno en América Latina. En J. Lanzaro, Tipos de Presidencialismo y Coaliciones Políticas en America Latina. (págs. 15-49). Buenos Aires: CLACSO.

LEVITSKY, Steven. (2018). Peru: The Institutionalization of Politics without Parties. En S. Mainwaring, Party Systems in Latin America: Institutionalization, Decay, and Collapse (págs. 326-356). Cambridge: Cambridge University Press.

LEVITSKY, Steven., \& Cameron, M. (2003). Democracy without parties? Political parties and regimen change in Fujimori's Peru. Latin American Politics and Society, 3(45), 1-33.

LEVITSKY, Steven., LOXTON, J., \& VAN DYCK, Brandon. (2016). Introduction: Challenges of party-building in Latin America. En S. L, J. L, B. VD, \& D. Jorge, Challenges of Party-Building in Latin America (págs. 1-51). Cambridge: Cambridge University Press.

LEVITT, Barry. (2012). Power in the Balance. Presidents, Parties and Legislatures un Peru and Beyond. Indiana: University of Notre Dame Press.

LINZ, Juan José. (1990). The perils of presidentialism. Journal of democracy, 1(1), 51-69.

LINZ, Juan José. (1999). Las restricciones temporales de la democracia. En S. A., \& S. J., Tiempo y democracia. Caracas: Editoriales Nueva Sociedad.

LINZ, Juan José, \& STEPAN, A. (1994). Problems of Democratic Transition and Consolidation: Southern. Baltimore: Johns Hopkins. 
LINZ, Juan José, \& VALENZUELA, Arturo. (1994). The breackdown of democracies. Baltimore: Johns Hopkins university Press.

LLANOS, Margarita. (2003). El bicameralismo en América Latina. Anuario de Derecho Constitucional Latinoamericano. Ciudad de México: Instituto de Investigaciones Jurídicas, UNAM.

MAINWARING, Scott. (1993). Presidentialism, Multipartism, and Democracy: The Difficult Combination. Comparative Political Studies, 26(2), 198-228.

MAINWARING, Scott. (1999). Rethinking Party Systems in the Third Wave of Democratization: The Case of Brazil. Palo Alto: Stanford University Press.

MAINWARING, Scott., \& SCULLY, Timothy. (1995). Building Democratic Institutions: Party Systems in Latin America. Palo Alto: Stanford University Press.

MAINWARING, Scott., \& SHUGART, Matthew. (1997). Presidentialism and democracy in Latin America. Cambridge: Cambridge University Press.

MELÉNDEZ, Carlos. (2006). Partidos y outsiders. El proceso electoral peruano de 2006. Desafíos, 1, 40-68.

MELÉNDEZ, Carlos. (2012). Partidos inesperados. La institucionalización del sistema de partidos en un escenario de post colapso partidario. Perú 2001-2011. Lima: Friedrich Ebert Stiftung.

MORGENSTERN, Scott. (2004). Patterns of Legislative Politics: Roll-Call Voting in Latin America and the United States. Cambridge: Cambridge University Press.

MORGENSTERN, S., NEGRI, J. J., \& PÉREZ-LIÑÁN, Anibal. (2009). La oposición parlamentaria en regímenes presidenciales: el caso latinoamericano. Jornada sobre el Parlamento y democracia en la realidad politica Iberoamericana (págs. 1-44). Zaragoza: Fundación Manuel Giménez Abad de Estudios Parlamentarios y del Estado Autonómico.

NEGRETTO, Jorge. (2006). Minority presidents and democratic performance. Latin American Politics and Society, 48(3), 63-92.

PAYNE, Michael. (2006). El equilibrio de poder entre el Ejecutivo y el Legislativo: papel de la Constitución y los partidos políticos. En M. Payne, D. Zovatto, \& M. Mateo Díaz, La política importa. Democracia y desarrollo en América Latina. . Washington: BID e IDEA.

RENIU, Josep María, \& ALBALA, José María. (2012). Los gobiernos de coalición y su incidencia sobre los presidencialismos latinoamericanos: el caso del Cono Sur. Los gobiernos de coalición y su incidencia sobre los presidencialismos(26), 161-214.

RUBIO CORREA, Marcial. (1999). Estudio de la Constitución Política de 1993. Tomo IV. Lima: Pontificia Universidad Católica del Perú.

SAMUELS, David, \& SHUGART, Matthew Scott (2010). Presidents, Parties, and Prime Ministers: How the Separation of Powers Affects Party Organization and Behavior. Cambridge: Cambridge University Press.

SÁNCHEZ, Francisco., NOLTE, Detlef., \& LLANOS, Margarita. (2005). Bicameralismo, Senados y senadores en el Cono Sur latinoamericano. Barcelona: Publicacions del Parlament de Catalunya.

SARTORI, Giovanni. (1966). Opposition and Control: Problems and Prospects. Government and Opposition, $1(1)$.

SEIFERT BONIFAZ, Manuel. (2014). Colapso de los partidos nacionales y auge de los partidos regionales. Lima: Pontificia Universidad Católica del Perú.

STEPAN, Alfred., \& SKACH, Cindy. (1993). Constitutional Frameworks and Democratic Consolidation: Parliamentarianism versus Presidentialism. World Politics, 46(1), 1-22.

STRØM, Kaare. (1984). Minority Governments in Parliamentary Democracies: The Rationality of NonWinning Cabinet Solutions. Comparative Political Studies, 199-227.

STRØM, Kaare. (1990). Minority Governement and Majority Rule. Cambridge: Cambridge University Press. 
TANAKA, Martín., \& VERA, Rojas. (2010). La dinámica 'neodualista' de una democracia sin sistema de partidos: La situación de la democracia en el Perú. Revista de Ciencia Política, 30(1), 87-114.

TANAKA, Martín., Villagarcia, Paolo Sosa, \& Puémape, Félix. (2016). ¿Una elección atípica? En F. Tuesta Soldevilla, Perú : elecciones 2016 : un país dividido y un resultado inesperado (págs. 259-285). Lima: Pontificia Universidad Católica del Perú, Fondo Editorial.

VAIRO, Daniela. (2016). Lealtad Democrática de los Partidos de Oposición. Revista de Ciências Sociais, 62(1), 1-35.

ZELAZNIK, Javier. (2002). The Building Of Coalitions in the Presidential Systems Of Latin America: An Inquiry into the Political Conditions of Governability. Tesis de Doctorado. Essex: University of Essex.

Notas

$1 \quad$ A partir de 2011 el Congreso de la República amplía sus curules de 120 a 130.

2 Cálculos elaborados con base en los datos disponibles de la Presidencia de la República.

3 Ana Jara durante el gobierno de Humala, Fernando Zavala con PPK y Salvador Del Solar y Pedro Cateriano con Vizcarra.

4 Cálculo elaborado con base en los datos disponibles de la Presidencia de la República y contabilizando a los diputados presentes en la votación.

5 De hecho las interpelaciones fueron escasamente empleadas como mecanismo de rendición de cuentas y cese de ministros, contabilizándose, para el período 2006-2020, 9 interpelaciones durante la segunda presidencia de García, 14 durante con Humala, 4 con Kuczynski y 5 con Vizcarra.

6 Cálculo propio realizado con base en datos de la Presidencia de la República. Corvetto (2014) muestra datos similares para el período de 1980 a 2011, a excepción de los dos mandatos de Alan García.

7 Fuerza Popular obtuvo 15 escaños. Destacable igualmente la debilidad parlamentaria de Vizcarra, ya que Somos Perú se situó en apenas 11 curules.

8 Sin embargo, no se trató de una coalición donde se repartieran carteras ministeriales y acción de gobierno, siguiendo las denominaciones habituales de Strøm (1990); Chasquetti (2008) y Albala (2009).

9 De hecho, es práctica habitual la mera amenaza del uso de la moción de censura para que se produzcan salidas anticipadas de ministros o primeros ministros.

10 Kuczynski y Vizcarra, quienes son analizados en el presente estudio; y Manuel Merino y Francisco Sagasti desde noviembre de 2020. 